\title{
On solutions of the singular minimal surface equation
}

\section{Ulrich Dierkes ${ }^{1}$}

Dedicated to Anthony Tromba on his seventy-fifth birthday

Received: 21 March 2018 / Accepted: 19 July 2018 / Published online: 21 August 2018

(c) Fondazione Annali di Matematica Pura ed Applicata and Springer-Verlag GmbH Germany, part of Springer Nature 2018

\section{Abstract}

Results of Bernstein type are proven for supersolutions of the singular minimal surface equation when $\alpha<0$. In particular the non-existence of "entire" minimal graphs in hyperbolic space is shown. In addition we construct a foliation of $\mathbb{R}^{n} \times \mathbb{R}^{+}$consisting of minimizing surfaces, and solve a Dirichlet problem for the singular minimal surface equation.

Keywords Bernstein-type theorems · Entire supersolutions · Minimal surfaces · Dirichlet problem

Mathematics Subject Classification 35A15 - 35B08 - 35J75 - 49Q05 - 49Q10 - 49Q20 . $53 \mathrm{~A} 10 \cdot 53 \mathrm{C} 42$

\section{Introduction}

In this paper, we are interested in the singular minimal surface equation (in short: s. m. s.e.)

$$
\operatorname{div}\left(\frac{D u}{\sqrt{1+|D u|^{2}}}\right)=\frac{\alpha}{u \sqrt{1+|D u|^{2}}} \text { in } \mathbb{R}^{n},
$$

where $\alpha \in \mathbb{R}$ is some real number and $u: \mathbb{R}^{n} \rightarrow \mathbb{R}$ denotes a differentiable function. In particular we here address the question of existence and non-existence of entire, i.e. on all of $\mathbb{R}^{n}$ defined (super-) solutions in cases where $\alpha<0$. As we show in the discussion to follow, the global behaviour of solutions of $(*)$ crucially depends on the sign of $\alpha$, in fact it is completely different in the three cases $\alpha<0, \alpha=0, \alpha>0$. In addition we also solve the Dirichlet problem for the s.m.s.e. (*) on interior mean convex regions $\Omega \subset \mathbb{R}^{n}$.

Obviously, if $\alpha=0$ then equation ( $*$ ) reduces to the classical minimal surface equation, which has only trivial entire solutions (affine functions) for $2 \leq n \leq 7$ and nonlinear solutions for all $n \geq 8$, see the fundamental paper by Bombieri-De Giorgi-Giusti [4].

$\triangle$ Ulrich Dierkes

ulrich.dierkes@uni-due.de

1 Fakultät für Mathematik, Universität Duisburg-Essen, Thea-Leymann-Straße 9, 45127 Essen, Germany 
Now if $\alpha>0$ there exist entire solutions of the s. m. s. e. in all dimensions $n \geq 2$, e.g. Lipschitz (weak)-solutions $c_{n}^{\alpha}(x)=\sqrt{\frac{\alpha}{n-1}}\|x\|$ and also smooth rotational symmetric solutions, $\mathrm{cp}$. the papers by Keiper [17], Dierkes [6] and Lopez [19]. However, if $\alpha+n$ is suitably small (i.e. $\alpha+n<4+\sqrt{\frac{2}{n+\alpha}}$ ) no stable entire solution can exist (see [7,8,10]), nor are there entire solutions of $(*)$ which are of sublinear growth (independent of $n$ ), see the work of Dierkes and Tennstädt [12]. Recall that "stability" requires that the second-variation $\delta^{2} E_{\alpha}$ is nonnegative, where $E_{\alpha}$ denotes the " $\alpha$-energy" or " $\alpha$-area"-functional

$$
E_{\alpha}(u)=\int u^{\alpha} \sqrt{1+|D u|^{2}} \mathrm{~d} x
$$

and that $(*)$ is the Euler equation for $E_{\alpha}$.

Alternatively equation $(*)$ can also be regarded as an equation of mean curvature type, the mean curvature $H$ with respect to the upper normal being given by

$$
H(u, D u)=\frac{\alpha}{u \sqrt{1+|D u|^{2}}} .
$$

There are several Bernstein-type results for equations of mean curvature type, starting with the celebrated result by Bernstein [3] himself, according to which no graph with mean curvature $\geq H_{0}>0$ can exist on a ball of radius $>\frac{1}{H_{0}}$, for improvements see Finn [13,14], Heinz [16] and Usami [20]. However, the class comprised and considered in these papers excludes cases like in $(*)$, where the mean curvature depends on both $u$ and $D u$. We prove the following

Theorem 1.1 Let $\alpha<0$. Then any $C^{2}$-function $u: \mathbb{R}^{n} \rightarrow \mathbb{R}$ which is an entire supersolution of $(*)$, i.e.

$$
\operatorname{div}\left(\frac{D u}{\sqrt{1+|D u|^{2}}}\right) \leq \frac{\alpha}{u \sqrt{1+|D u|^{2}}} \text { on } \mathbb{R}^{n}
$$

is nowhere positive. In particular there are no nonnegative entire $C^{2}$-solutions $u: \mathbb{R}^{n} \rightarrow$ $\mathbb{R}_{0}^{+}=\{t \geq 0\}$ of inequality (1.1).

Clearly, any constant function $u \equiv c<0$ satisfies inequality (1.1). On the other hand applying the same reasoning to both $u$ and $(-u)$, we obtain the following Bernstein-type result

Corollary 1.2 Let $\alpha<0$, then there is no entire $C^{2}$-solution $u: \mathbb{R}^{n} \rightarrow \mathbb{R}$ of the s. m.s.e. $(*)$.

\section{Remark 1.3}

(i) Non-existence of positive, entire $C^{2}$-solutions of the s.m.s.e. $(*)$ in the two-dimensional case has recently been proved by Lopez [19] in a forthcoming paper.

(ii) For $\alpha=-n$ equation ( $*$ ) arises in the study of minimal hypersurfaces in hyperbolic space $\mathbb{H}^{n+1}(-1)$ modelled by the upper half space

$$
\mathbb{R}^{n} \times \mathbb{R}^{+}:=\left\{\left(x, x_{n+1}\right) \in \mathbb{R}^{n} \times \mathbb{R} ; x_{n+1}>0\right\}
$$

with the hyperbolic metric

$$
d s^{2}=\frac{1}{x_{n+1}^{2}}\left(d x^{2}+d x_{n+1}^{2}\right)
$$

of curvature -1 . 
Thus we have

Corollary 1.4 There is no entire $C^{2}$-minimal graph in $\mathbb{H}^{n+1}(-1)$ for all $n \geq 2$.

Note that here we have used the expression "entire" in a strictly euclidean sense, meaning that $u$ is defined on all of $\mathbb{R}^{n}$. Indeed, the existence of complete minimal graphs in $\mathbb{H}^{n+1}(-1)$ with asymptotic boundary at "infinity" $\partial \Omega \subset \mathbb{R}^{n} \times\{0\}$, that is, solutions $u \in C^{2}(\Omega) \cap C^{0}(\bar{\Omega})$ of (*) with $\alpha=-n$ and $\Omega \subset \mathbb{R}^{n}$ a mean convex domain, $u=0$ on $\partial \Omega$, was shown in the papers by Anderson [1] and Lin [18], see also our Theorem 1.6.

There is a "parametric" version of Theorem 1.1. To give a clear statement, consider a $C^{2}$ submanifold $M \subset \mathbb{R}^{n} \times \mathbb{R}^{+}, \mathbb{R}^{+}=\{t>0\}$ and let $\mathcal{E}_{\alpha}$ denote the parametric counterpart of $E_{\alpha}$, i.e.

$$
\mathcal{E}_{\alpha}(M):=\int_{M} x_{n+1}^{\alpha} d \mathcal{H}_{n},
$$

where $\mathcal{H}_{n}$ stands for the $n$-dimensional Hausdorff-measure. One readily verifies that the first variation $\delta \mathcal{E}_{\alpha}(M, X)$ vanishes for all compactly supported vectorfields $X$, if and only if the mean curvature with respect to the unit normal $v=\left(v_{1}, \ldots, v_{n+1}\right)$ is given by

$$
H(x)=\alpha x_{n+1}^{-1} v_{n+1}
$$

(cp.[10, chap.3.2]). In this case $M$ is called " $\alpha$-stationary". Observe that the vertical planes

$$
E:=\left\{\left(x, x_{n+1}\right) \in \mathbb{R}^{n} \times \mathbb{R}^{+} ; a \cdot x=\mathrm{const}\right\}
$$

where $a \in \mathbb{R}^{n}$ are $\alpha$-stationary hypersurfaces for arbitrary $\alpha \in \mathbb{R}$. By a similar argument as in Theorem 1.1 we can prove

Theorem 1.5 Let $\alpha<0$, and put $\mathbb{R}_{0}^{+}=\{t \geq 0\}$. There are no $\alpha$-stationary $C^{2}$-submanifolds $M$ in $\mathbb{R}^{n} \times \mathbb{R}^{+}$with $\partial M \cap\left(\mathbb{R}^{n} \times \mathbb{R}_{0}^{+}\right)=\emptyset$.

It is well known and easily verified, that every upper halfsphere

$$
u(x)=\sqrt{R^{2}-\left\|x-x_{0}\right\|^{2}}, x_{0} \in \mathbb{R}^{n}
$$

solves the minimal surface equation in $\mathbb{H}^{n+1}(-1)$ on balls $B_{R}\left(x_{0}\right)$, i.e. we have

$$
\operatorname{div}\left(\frac{D u}{\sqrt{1+|D u|^{2}}}\right)=\frac{-n}{u \sqrt{1+|D u|^{2}}} \quad \text { in } B_{R}\left(x_{0}\right),
$$

and $u=0$ on $\partial B_{R}\left(x_{0}\right)$.

Furthermore it was shown in [18] that each halfsphere is locally (hyperbolic-)area minimizing in $\mathbb{H}^{n+1}(-1)$. A crucial point in the present paper is the construction of classical $C^{2}\left(B_{R}\right) \cap$ $C^{0, \frac{1}{2}}\left(\bar{B}_{R}\right)$ solutions of the s.m. s.e. $(*)$ for arbitrary $n \geq 2$ and any $\alpha<0$, which generalizes the upper hemisphere solution described above. Moreover, we show that these solutions give rise to a whole foliation of $\mathbb{R}^{n} \times \mathbb{R}^{+}$by singular minimal surfaces. Using a variant of a method due to Bombieri-De Giorgi-Giusti [4] and the present author [6], we then show that each element of the foliation is infact $\mathcal{E}_{\alpha}$-minimizing in a very general sense, see Theorem 2.1. Combining these solutions with E. Hopfs' boundary point lemma, we can prove Theorem 1.1 in a classical way.

As a further consequence of the minimal foliation constructed in Theorem 2.1 and the general theory of quasilinear elliptic equations (see for example Gilbarg-Trudinger [15]), we find the following 
Theorem 1.6 Suppose $\Omega \subset \mathbb{R}^{n}$ is a bounded $C^{2}$-domain which is inward mean convex, i.e. the inward mean curvature of $\partial \Omega$ is nonnegative and let $\alpha<0$. Then the Dirichlet problem

$$
\begin{aligned}
\operatorname{div}\left(\frac{D u}{\sqrt{1+|D u|^{2}}}\right) & =\frac{\alpha}{u \sqrt{1+|D u|^{2}}} \text { in } \Omega, \\
u & =0 \text { on } \partial \Omega
\end{aligned}
$$

has a unique positive solution $u \in C^{2}(\Omega) \cap C^{0}(\bar{\Omega})$.

When $\alpha=-n$ and $u \in C^{2}(\Omega) \cap C^{0}(\bar{\Omega})$ is a solution of the Dirichlet problem (1.2), thenas already mentioned above- $\operatorname{graph}(u)$ is a complete minimal hypersurface in hyperbolic space $\mathcal{H}^{n+1}(-1)$ with asymptotic boundary $\partial \Omega \times\{0\}$ at infinity. For this case problem (1.2) was solved by Anderson [1] and-with a different argument-by Lin [18]. Invoking Theorem 2.1 we may apply a similar argument as given in [18] to prove Theorem 1.6, for details see Sect. 3.

The Dirichlet problem associated with the s. m. s.e. when $\alpha>0$ and $\Omega \subset \mathbb{R}^{n}$ is mean convex has been considered and solved in Dierkes [5,9] and Dierkes-Huisken [11].

\section{Functions of least weighted gradient and boundaries of least $\alpha$-area}

In the following $\mathbb{R}^{+}=\{t>0\}$ stands for the positive real numbers and we work in the open half space $\mathbb{R}^{n} \times \mathbb{R}^{+}=\left\{x=\left(\hat{x}, x_{n+1}\right) ; \hat{x} \in \mathbb{R}^{n}, x_{n+1}>0\right\}$. Also $\alpha<0$ denotes a negative real number. If $A \subset \mathbb{R}^{n} \times \mathbb{R}^{+}$is an open set and $f \in B V_{\text {loc }}(A)$, we say that $f$ has "least $\alpha$-weighted gradient" (or simply "least $\alpha$-gradient") in $A$, if

$$
\int_{K} x_{n+1}^{\alpha}|D f| \leq \int_{K} x_{n+1}^{\alpha}|D(f+g)|
$$

for every function $g \in B V_{\text {loc }}(A)$ with compact support $K \subset A$.

Moreover, a set $C \subset A$ has boundary of "least $\alpha$-area" with respect to $A$, if the characteristic function $\varphi_{C} \in B V_{\mathrm{loc}}(A)$ and has least $\alpha$-gradient in $A$.

We have the following

Theorem 2.1 Let $n \geq 2$ and $\alpha<0$. There exists a foliation of $\mathbb{R}^{n} \times \mathbb{R}^{+}$determined by concave rotational symmetric functions $v_{\lambda}=v_{\lambda}(\hat{x}): B_{\lambda}(0) \rightarrow \mathbb{R}^{+}, \lambda>0$ arbitrary, $v_{\lambda}(0)=\lambda \cdot v_{1}(0)$ and $D v_{\lambda}(0)=0$. Furthermore, for each $\lambda>0$ the functions $v_{\lambda} \in C^{\omega}\left(B_{\lambda}(0)\right) \cap C^{0}\left(\bar{B}_{\lambda}(0)\right)$, solve the Dirichlet problem

$$
\begin{aligned}
\operatorname{div}\left(\frac{D v_{\lambda}}{\sqrt{1+\left|D v_{\lambda}\right|^{2}}}\right) & =\frac{\alpha}{v_{\lambda} \sqrt{1+\left|D v_{\lambda}\right|^{2}}} \text { in } B_{\lambda}(0), \\
v_{\lambda} & =0 \text { on } \partial B_{\lambda}(0)
\end{aligned}
$$

and satisfy $\left|D v_{\lambda}(\hat{x})\right| \rightarrow \infty$ as $\hat{x} \rightarrow \partial B_{\lambda}(0)$.

If $\alpha<-1$ the functions $v_{\lambda}$ belong to $C^{0, \frac{1}{2}}\left(\bar{B}_{\lambda}\right)$. Finally the subgraphs

$$
V_{\lambda}:=\left\{\left(\hat{x}, x_{n+1}\right) \in \mathbb{R}^{n} \times \mathbb{R}^{+} ; \hat{x} \in B_{\lambda}(0), 0<x_{n+1}<v_{\lambda}(\hat{x})\right\}, \text { for } \lambda>0,
$$

have boundaries of least $\alpha$-area in $\mathbb{R}^{n} \times \mathbb{R}^{+}$. In particular the functions $v_{\lambda}$ minimize locally the (nonparametric) $\alpha$-area

$$
E_{\alpha}(u)=\int u^{\alpha} \sqrt{1+|D u|^{2}} \text { in } B V\left(B_{\lambda}(0)\right) .
$$


The proof of Theorem 2.1 is as follows: We show that any function $f$ of least $\alpha$-gradient has (sub-) level sets of least $\alpha$-area (Lemma 2.3). Moreover, any solution $f \in H_{1, \text { loc }}^{1}(A)$ of the equation

$$
\int_{A} x_{n+1}^{\alpha}|D f|^{-1} D f D \varphi \mathrm{d} x=0, \quad \forall \varphi \in C_{c}^{\infty}(A)
$$

where $D f=\left(D_{1} f, \ldots, D_{n} f\right)$ has least $\alpha$-gradient (Lemma 2.2). Here a locally integrable function $f$ is of class $H_{1, \text { loc }}^{1}(A)$, if its weak derivative is again locally integrable i.e. in $L_{1, \text { loc }}(A)$. Finally a function $f(x)=F(u, v)$ of the two variables

$$
u:=|\hat{x}|=\left\{x_{1}^{2}+\ldots+x_{n}^{2}\right\}^{\frac{1}{2}}, v:=\left(x_{n+1}^{2}\right)^{\frac{1}{2}}=x_{n+1}
$$

is constructed, which is of least $\alpha$-gradient and has level sets with the properties described in Theorem 2.1.

The following Lemmata are analogues to the results in [6], Lemmas 1 and 3 for the case of positive $\alpha$, so we skip over the proofs referring to the arguments in the paper loc. cit.

Lemma 2.2 Let $A \subset \mathbb{R}^{n} \times \mathbb{R}^{+}$be an open set and $\mathcal{N}$ be some closed set with $\mathcal{H}_{n}(\mathcal{N})=0$. Suppose $f \in H_{1, \text { loc }}^{1}(A)$ satisfies

$$
\mathcal{H}_{n+1}(\{x \in A ;|D f(x)|=0\})=0,
$$

and

$$
\int_{A} x_{n+1}^{\alpha}|D f|^{-1} D f \cdot D \varphi \mathrm{d} x=0
$$

for every $\varphi \in C_{c}^{1}(A-\mathcal{N})$. Then $f$ has least $\alpha$-gradient in $A$.

For the proof we refer to Lemma 1 in [6]. Observe that here $\mathbb{R}^{+}$stands for the positive real numbers, while $\mathbb{R}^{+}:=\{t \geq 0\}$ in [6].

Lemma 2.3 Let $A \subset \mathbb{R}^{n} \times \mathbb{R}^{+}$and $f \in B V_{\text {loc }}(A)$ be a function of least $\alpha$ gradient in $A$. Then every nonempty level set

$$
E_{\lambda}:=\{x \in A ; f(x) \geq \lambda\}
$$

has an oriented boundary of least $\alpha$ area in $A$.

The proof follows from the coarea formula and some approximation device analogously to Lemma 3 in [6].

\section{Proofs}

It follows from Sect. 2 that we have to find a function $f$ of least $\alpha$-gradient in $\mathbb{R}^{n} \times \mathbb{R}^{+}$ which has level sets given by functions $v_{\lambda}: B_{\lambda}(0) \rightarrow \mathbb{R}^{+}$with the properties described in Theorem 2.1. Since we are looking for rotational symmetric solutions, it is convenient to introduce the variables

$$
\left\{\begin{array}{l}
u:=\left(x_{1}^{2}+\cdots+x_{n}^{2}\right)^{\frac{1}{2}}=|\hat{x}|, \text { and } \\
v:=\left(x_{n+1}^{2}\right)^{\frac{1}{2}}=x_{n+1},
\end{array}\right.
$$


where $x=\left(\hat{x}, x_{n+1}\right)=\left(x_{1}, \ldots, x_{n}, x_{n+1}\right) \in \mathbb{R}^{n} \times \mathbb{R}^{+}, \mathbb{R}^{+}=\{t>0\}$ and consider functions $f(x):=F(u(x), v(x))$ where $F: \mathbb{R}_{0}^{+} \times \mathbb{R}^{+} \rightarrow \mathbb{R}, \mathbb{R}_{0}^{+}:=\{t \geq 0\}$. Let the open set $\tilde{\Omega} \subset \mathbb{R}^{+} \times \mathbb{R}^{+}$correspond to $\Omega \subset \mathbb{R}^{n} \times \mathbb{R}^{+}$under the transformation (3.1), then $f=f(x) \in C^{2}(\Omega),|\nabla f| \neq 0$, satisfies the Euler equation

$$
\sum_{i=1}^{n+1} D_{i}\left\{x_{n+1}^{\alpha} \frac{D_{i} f}{|D f|}\right\}=0 \text { in } \Omega
$$

if and only if $F=F(u, v)$ fulfils

$$
\frac{\partial}{\partial u}\left\{\frac{u^{n-1} v^{\alpha} F_{u}}{|\nabla F|}\right\}+\frac{\partial}{\partial v}\left\{\frac{u^{n-1} v^{\alpha} F_{v}}{|\nabla F|}\right\}=0 \text { in } \tilde{\Omega},
$$

where we have put

$$
F_{u}=\frac{\partial F}{\partial u}, F_{v}=\frac{\partial F}{\partial v} \text { and }|\nabla F|^{2}=F_{u}^{2}+F_{v}^{2} .
$$

(3.3) in turn is equivalent to

$$
F_{v}^{2} F_{u u}-2 F_{u} F_{v} F_{u v}+F_{u}^{2} F_{v v}+\left(F_{u}^{2}+F_{v}^{2}\right)\left[p \frac{F_{u}}{u}+\alpha \frac{F_{v}}{v}\right]=0 \text { in } \tilde{\Omega}
$$

with $p:=n-1$.

The unknown function $F=F(u, v)$ will now be constructed from its level sets $\{F=$ const $\}$. Along a regular level curve, we have

$$
d F=F_{u} d u+F_{v} d v=0,
$$

which yields when applied to (3.4) the equation

$$
u^{\prime \prime} v^{\prime}-v^{\prime \prime} u^{\prime}+\left\{\left(u^{\prime}\right)^{2}+\left(v^{\prime}\right)^{2}\right\}\left[\alpha \frac{u^{\prime}}{v}-p \frac{v^{\prime}}{u}\right]=0,
$$

where $\left(u(t), v(t) \in \mathbb{R}^{+} \times \mathbb{R}^{+}, t \in \mathbb{R}\right.$, is a twice differentiable parametrization of the level curve.

We introduce the coordinates

$$
\left\{\begin{array}{l}
\varphi:=\operatorname{arctg}\left(\frac{v}{u}\right), \text { the "polar angle" and } \\
\theta:=\operatorname{arctg}\left(\frac{v^{\prime}}{u^{\prime}}\right), \text { the "tangent angle" }
\end{array}\right.
$$

which are both invariant under homotheties

$$
(u(t), v(t)) \rightarrow \lambda(u(t), v(t)) \text { for } \lambda>0 .
$$

From (3.6) we infer the relations

$$
\varphi^{\prime}=\frac{v^{\prime} u-u^{\prime} v}{u^{2}+v^{2}} \quad \text { and } \quad \theta^{\prime}=\frac{v^{\prime \prime} u^{\prime}-v^{\prime} u^{\prime \prime}}{\left(u^{\prime}\right)^{2}+\left(v^{\prime}\right)^{2}},
$$

whereas from

$$
\begin{array}{ll}
u=\sqrt{u^{2}+v^{2}} \cos \varphi & , v=\sqrt{u^{2}+v^{2}} \sin \varphi \\
u^{\prime}=\sqrt{\left(u^{\prime}\right)^{2}+\left(v^{\prime}\right)^{2}} \cos \theta, & v^{\prime}=\sqrt{\left(u^{\prime}\right)^{2}+\left(v^{\prime}\right)^{2}} \sin \theta
\end{array}
$$


we have

$$
\varphi^{\prime}=\left\{\frac{\left(u^{\prime}\right)^{2}+\left(v^{\prime}\right)^{2}}{u^{2}+v^{2}}\right\}^{\frac{1}{2}} \sin (\theta-\varphi) .
$$

On the other hand (3.5) applied to $\theta^{\prime}$ gives

$$
\theta^{\prime}=\alpha \frac{u^{\prime}}{v}-p \frac{v^{\prime}}{u}=\left\{\frac{\left(u^{\prime}\right)^{2}+\left(v^{\prime}\right)^{2}}{u^{2}+v^{2}}\right\}^{\frac{1}{2}}\left\{\alpha \frac{\cos \theta}{\sin \varphi}-p \frac{\sin \theta}{\cos \varphi}\right\}
$$

which together with (3.7) yields the formula

$$
2[\alpha \cos \theta \cos \varphi-p \sin \theta \sin \varphi] \varphi^{\prime}-[\sin 2 \varphi \sin (\theta-\varphi)] \theta^{\prime}=0 .
$$

Whence the level curves of the function $F$ are described by the ordinary planar system

$$
\begin{aligned}
& \frac{\mathrm{d} \varphi}{\mathrm{d} t}=\sin (2 \varphi) \sin (\theta-\varphi)=: \Phi(\varphi, \theta) \\
& \frac{\mathrm{d} \theta}{\mathrm{d} t}=2 \alpha \cos \theta \cos \varphi-2 p \sin \theta \sin \varphi=: \Psi(\varphi, \theta)
\end{aligned}
$$

which we analyse according to Poincaré-Bendixson theory in the rectangle $(\varphi, \theta) \in\left[0, \frac{\pi}{2}\right] \times$ $\left[-\frac{\pi}{2}, 0\right]$.

Since $\Phi(\varphi, \theta)=\sin (2 \varphi) \sin (\theta-\varphi) \neq 0$ for all $(\varphi, \theta) \in\left(0, \frac{\pi}{2}\right) \times\left(-\frac{\pi}{2}, 0\right)$ system (3.8) has no singular points in the open rectangle $\left(0, \frac{\pi}{2}\right) \times\left(-\frac{\pi}{2}, 0\right)$. Indeed, the only singular points in the closed interval $\left[0, \frac{\pi}{2}\right] \times\left[-\frac{\pi}{2}, 0\right]$ are the points $\left(\frac{\pi}{2}, 0\right)$ and $\left(0,-\frac{\pi}{2}\right)$. We distinguish between the following cases:

First case. The singular point $\left(\frac{\pi}{2}, 0\right)$ is a saddle point and the linearized system has matrix

$$
\left(\begin{array}{cc}
2 & 0 \\
-2 \alpha & -2 p
\end{array}\right)
$$

The direction of the stable manifolds is $\pm \infty$, while the two unstable manifolds have directions

$$
t_{1}:=\frac{-\alpha}{1+p}=\frac{-\alpha}{n}>0 .
$$

Second case. The singular point $\left(\varphi_{0}, \theta_{0}\right)=\left(0,-\frac{\pi}{2}\right)$ is a stable node and the linearized system (3.8) has the matrix

$$
\left(\begin{array}{cc}
-2 & 0 \\
2 p & 2 \alpha
\end{array}\right)
$$

If $-1<\alpha<0$ or $\alpha<-1$ the principal direction is $\pm \infty$ or $t_{2}:=\frac{-p}{1+\alpha}>0$, respectively; while the exceptional directions are

$$
\left.t_{3}:=\frac{-p}{1+\alpha}<0 \quad(\text { for }-1<\alpha<0) \text { and } \pm \infty \quad \text { (if } \alpha<-1\right) \text { respectively. }
$$

Finally, if $\alpha=-1$ the node is improper and all stable manifolds have direction $\pm \infty$.

Lemma 3.1 Let $\alpha<0$ and $p=n-1 \geq 1$. There exists a trajectory $\gamma(t)=(\varphi(t), \theta(t)), t \in$ $(-\infty, \infty)$ which solves the system (3.8) and has the following properties:

(i) $\gamma(-\infty)=\left(\frac{\pi}{2}, 0\right), \gamma(\infty)=\left(0,-\frac{\pi}{2}\right)$

(ii) $\gamma(t) \in\left(0, \frac{\pi}{2}\right) \times\left(-\frac{\pi}{2}, 0\right)$ for all $t \in(-\infty, \infty)$.

(iii) $\frac{\mathrm{d} \varphi}{\mathrm{d} t}<0$ and $\frac{\mathrm{d} \theta}{\mathrm{d} t}<0$ for all $t \in(-\infty, \infty)$. 
In particular the integral curve $\gamma$ has a nonparametric representation $\theta=\Theta(\varphi)$ for some differentiable function $\Theta:\left(0, \frac{\pi}{2}\right) \rightarrow\left(-\frac{\pi}{2}, 0\right)$ with $\Theta(0)=-\frac{\pi}{2}, \quad \Theta\left(\frac{\pi}{2}\right)=0$ and $\frac{\mathrm{d} \Theta}{\mathrm{d} \varphi}>0$.

Proof of Lemma 3.1 Consider the rectangle

$$
R:=\left\{(\varphi, \theta) ; 0<\varphi<\frac{\pi}{2} \text { and }-\frac{\pi}{2}<\theta<0\right\}
$$

with the sides

$$
\begin{aligned}
\ell_{1} & :=\left\{0<\varphi<\frac{\pi}{2}, \quad \theta=0\right\}, \\
\ell_{2} & :=\left\{\varphi=\frac{\pi}{2}, \quad-\frac{\pi}{2}<\theta<0\right\}, \\
\ell_{3} & :=\left\{0<\varphi<\frac{\pi}{2}, \quad \theta=-\frac{\pi}{2}\right\}, \text { and } \\
\ell_{4} & :=\left\{\varphi=0, \quad-\frac{\pi}{2}<\theta<0\right\} .
\end{aligned}
$$

By (3.8) we have on $\ell_{1}, \ell_{2}, \ell_{3}$ and $\ell_{4}$, respectively

$$
\begin{aligned}
& \Phi_{\mid \ell_{1}}=\sin (2 \varphi) \sin (-\varphi)<0, \quad \Psi_{\mid \ell_{1}}=2 \alpha \cos \varphi<0, \\
& \Phi_{\mid \ell_{2}}=0, \quad \Psi_{\mid \ell_{2}}=-2 p \sin \theta>0, \\
& \Phi_{\mid \ell_{3}}=-\sin (2 \varphi) \cos \varphi<0, \quad \Psi_{\mid \ell_{3}}=2 p \sin \varphi>0, \quad \text { and } \\
& \Phi_{\mid \ell_{4}}=0, \quad \Psi_{\mid \ell_{4}}=2 \alpha \cos \theta<0 .
\end{aligned}
$$

Therefore, the right-hand side $(\Phi(\varphi, \theta), \Psi(\varphi, \theta))$ of the system (3.8) at a regular boundary point of the rectangle $\partial R=\ell_{1} \cup \ell_{2} \cup \ell_{3} \cup \ell_{4}$ is directed into the closure $\bar{R}$ of the quadrilateral $R$, in fact in the interior along the sides $\ell_{1}$ and $\ell_{3}$. It follows from the preceding discussion of system (3.8) that one of the unstable manifolds, $\gamma(t):=(\varphi(t), \theta(t))$, starting at the saddle point $\left(\frac{\pi}{2}, 0\right)$ with direction $t_{1}=-\frac{\alpha}{n}>0$ into the interior of $R$ cannot leave $\bar{R}$ and hence approaches the stable node $\left(0,-\frac{\pi}{2}\right)$ with slope $t_{2}=-\frac{p}{1+\alpha}$ or $+\infty$. This implies (i) and (ii) of the Lemma. Since $\Phi(\varphi, \theta)<0$ on all of $R$ also $\frac{d \varphi}{d t}<0$ is obvious. For $t>-\infty$ "close to $-\infty$ " we conclude from the discussion above, that $\theta^{\prime}(t)<0$. We claim that $\theta^{\prime}(t)<0$ for all $t \in \mathbb{R}$. Indeed, suppose on the contrary that there is (by analyticity) a smallest $t_{0}>-\infty$ with $\theta^{\prime}\left(t_{0}\right)=0$ and $\theta^{\prime \prime}\left(t_{0}\right) \geq 0$. Then (3.8) yields the contradiction $\theta^{\prime \prime}\left(t_{0}\right)=-2 \alpha \cos \theta \sin \varphi \cdot \varphi^{\prime}\left(t_{0}\right)-2 p \sin \theta \cos \varphi \cdot \varphi^{\prime}\left(t_{0}\right)<0$, since $\alpha<0, \varphi^{\prime}\left(t_{0}\right)<0$ and $0<\varphi\left(t_{0}\right)<\frac{\pi}{2},-\frac{\pi}{2}<\theta\left(t_{0}\right)<0$.

Concluding we can write $t=T(\varphi)$ to denote the inverse function of $\varphi=\varphi(t)$ and $\theta=$ $\theta(t)=\theta(T(\varphi))=: \Theta(\varphi)$, where $\Theta:\left[0, \frac{\pi}{2}\right] \rightarrow\left[-\frac{\pi}{2}, 0\right]$ is monotonically increasing with $\Theta(0)=-\frac{\pi}{2}$ and $\Theta\left(\frac{\pi}{2}\right)=0$.

Proof of Theorem 2.1 Recalling the transformation (3.6)

$$
\varphi=\operatorname{arctg} \frac{v}{u} \quad \text { and } \quad \theta=\operatorname{arctg} \frac{v^{\prime}}{u^{\prime}}
$$

we see that $\gamma(t)=(\varphi(t), \theta(t))$ induces an analytic curve $\Gamma$ given by $(u(t), v(t))$ in the $(u, v)$-plane, which by virtue of Lemma 3.1 lies completely in the quadrant

$$
T=\{(u, v) ; u \geq 0, v \geq 0\} .
$$

Since $\varphi, \theta$ are invariant under homotheties, we obtain a "field" of curves $\Gamma_{\lambda}, \lambda>0$, which covers $T$ simply (quartercircles if $\alpha=-n$ ). 
Let $\Gamma_{1}$ be the curve issuing vertically from the point $(1,0)$. By Lemma 3.1 we may introduce $\varphi$ as a parameter on $\Gamma_{1}$ which leads to the representation for $\Gamma_{1}$

$$
u=u_{1}(\varphi), v=v_{1}(\varphi), \varphi \in\left[0, \frac{\pi}{2}\right],\left(u_{1}(\varphi)=\sin \varphi, v_{1}(\varphi)=\cos \varphi \text { if } \alpha=-n\right) .
$$

Alternatively, since $\theta \in\left(0,-\frac{\pi}{2}\right)$ for $\varphi \in\left(0, \frac{\pi}{2}\right)$ the curve $\Gamma_{1}$ may be written as graph $v=v_{1}(u), 0 \leq u \leq 1$ for some differentiable function $v_{1}$ with $v_{1}(0)>0, D v_{1}(0)=0$ and $D v_{1}(u) \rightarrow-\infty$ for $u \rightarrow 1$. In addition, since $\frac{\mathrm{d} \theta}{\mathrm{d} t}<0$, the representation $v_{1}$ must be a concave function.

Now any function $F=F(u, v)$ with $|\nabla F| \neq 0$, defined on the open set $\stackrel{\circ}{T}=\{u>0, v>0\}$ which has the curves $\Gamma_{\lambda}$ as level curves must satisfy Eq. (3.3). We choose the homogeneous function

$$
F(u, v):=\left(u^{2}+v^{2}\right) \cdot H\left(\operatorname{arctg} \frac{v}{u}\right), \quad \text { where }(u, v) \in \stackrel{\circ}{T},
$$

normalized by the requirement

$$
F\left(u_{1}(\varphi), v_{1}(\varphi)\right)=1 \text { for all } \varphi \in\left(0, \frac{\pi}{2}\right)
$$

whence

$$
H(\varphi)=\left(u_{1}^{2}(\varphi)+v_{1}^{2}(\varphi)\right)^{-1}, \quad \text { for } \varphi \in\left(0, \frac{\pi}{2}\right) \text { and } H(0)=1 .
$$

Clearly, $F=F(u, v)$ extends continuously onto the closure $T$ and is-by construction-an analytic solution of (3.4) in the interior $\stackrel{\circ}{T}$. Moreover, we have

$$
|\nabla F(u, v)|=\left(u^{2}+v^{2}\right)^{\frac{1}{2}}\left(4 H^{2}+H^{\prime 2}\right)^{\frac{1}{2}} \neq 0 \text { and } F\left(\Gamma_{\lambda}\right)=\lambda^{2}, \lambda>0 .
$$

On putting

$$
f(x):=F(u(x), v(x))
$$

we obtain a classical solution of Eq. (3.2) in the open set $\mathbb{R}^{n} \times \mathbb{R}^{+} \cap\{u \neq 0\}$ which has locally bounded gradient on $\mathbb{R}^{n} \times \mathbb{R}^{+}$. Hence we may apply Lemma 2.1 with $A:=\mathbb{R}^{n} \times \mathbb{R}^{+}$and $\mathcal{N}:=\left\{x \in \mathbb{R}^{n} \times \mathbb{R}_{0}^{+} ; u=0\right\}$, to conclude that $f=f(x)$ has least $\alpha$-gradient in $\mathbb{R}^{n} \times \mathbb{R}^{+}$. Recall that the level curves $\Gamma_{\lambda}, \lambda>0$ constitute a foliation of the quadrant $T=\{u, v \geq 0\}$ and that - by virtue of Lemma 3.1-each $\Gamma_{\lambda}$ gives rise to a function $v_{\lambda}=v_{\lambda}(\hat{x})$ defined on balls $\overline{B_{\lambda}(0)} \subset \mathbb{R}^{n}$ such that

$$
v_{\lambda} \in C^{1}\left(B_{\lambda}(0)\right) \cap C^{\infty}\left(B_{\lambda}(0)-\{0\}\right) \cap C^{0}\left(\overline{B_{\lambda}}\right) \text { and } v_{\lambda}=0 \text { on } \partial B_{\lambda}(0) .
$$

In particular the subgraph

$$
V_{\lambda}:=\left\{\left(\hat{x}, x_{n+1}\right) \in B_{\lambda}(0) \times \mathbb{R}^{+} ; x_{n+1}<v_{\lambda}(\hat{x})\right\}
$$

has an oriented boundary of least $\alpha$-area in $\mathbb{R}^{n} \times \mathbb{R}^{+}$. As in [2] one can then show that any function $v_{\lambda}$ locally minimizes the nonparametric $\alpha$-area

$$
E_{\alpha}(u):=\int u^{\alpha} \sqrt{1+|D u|^{2}} \quad \text { in } B V_{\mathrm{loc}}\left(B_{\lambda}(0)\right) .
$$

Therefore, the s.m. s.e. $(*)$ is satisfied classically in $B_{\lambda}-\{0\}$ and weakly in $B_{\lambda}(0)$; by elliptic regularity theory it follows that $v_{\lambda} \in C^{\omega}\left(B_{\lambda}(0)\right)$ and solves s.m.s.e. $(*)$ in the usual sense on the ball $B_{\lambda}(0)$. 
To conclude with the proof of Theorem 2.1 we finally show $\frac{1}{2}$-höldercontinuity of $v_{\lambda}$ on $\overline{B_{\lambda}(0)}$ if $\alpha<-1$. By the discussion preceding Lemma 3.1 the point $(\varphi, \theta)=\left(0,-\frac{\pi}{2}\right)$ is a stable node with principal direction $t_{2}=-\frac{p}{1+\alpha}>0$ and exceptional direction $\pm \infty$. Hence the separatrix $\gamma(t)$ in Lemma 3.1 must have direction $t_{2}$ at the point $\left(0,-\frac{\pi}{2}\right)$. Consequently we have the asymptotic expansion

$$
\theta=-\frac{\pi}{2}+t_{2} \varphi+o(\varphi) \text { as } \varphi \rightarrow 0^{+}
$$

while on the other hand

$$
\begin{aligned}
& \frac{v_{1}}{u_{1}}=\operatorname{tg} \varphi, \frac{v_{1}^{\prime}}{u_{1}^{\prime}}=\operatorname{tg} \theta \quad \text { i. e. } \\
& \frac{u_{1}^{\prime}}{u_{1}}=\frac{1}{\cos ^{2} \varphi} \cdot \frac{1}{\operatorname{tg} \theta-\operatorname{tg} \varphi}=\frac{\cos \theta}{\cos \varphi} \cdot \frac{1}{\sin (\theta-\varphi)} .
\end{aligned}
$$

Asymptotically we find

$$
\begin{aligned}
& \frac{u_{1}^{\prime}}{u_{1}} \sim-\cos \theta \sim-\cos \left(t_{2} \varphi-\frac{\pi}{2}\right)=-\sin \left(t_{2} \varphi\right)=-t_{2} \varphi+o(\varphi) \text { as } \varphi \rightarrow 0^{+} \\
& \text {whence } u_{1}(\varphi)=1-\frac{t_{2}}{2} \varphi^{2}+o\left(\varphi^{2}\right) \text { as } \varphi \rightarrow 0^{+} .
\end{aligned}
$$

But $v_{1}=u_{1} \cdot \operatorname{tg} \varphi$, so $v_{1}(\varphi)=\varphi+o(\varphi)$ as $\varphi \rightarrow 0^{+}$from which we conclude that $v_{1} \in C^{0, \frac{1}{2}}\left(\overline{B_{1}(0)}\right)$ and also $v_{\lambda} \in C^{0, \frac{1}{2}}\left(\overline{B_{\lambda}}\right)$.

Theorem 2.1 follows.

Proof of Theorem 1.1 Suppose by contradiction that a solution $u$ of inequality (1.1) exists for which $u\left(x_{0}\right)>0$ at some point $x_{0} \in \mathbb{R}^{n}$. Without loss of generality we can assume that $x_{0}=0$ and consider the symmetric minimizers $v_{\lambda}: \overline{B_{\lambda}(0)} \rightarrow \mathbb{R}^{+} \cup\{0\}$ with $\left|D v_{\lambda}(x)\right| \rightarrow \infty$ as $x \rightarrow \partial B_{\lambda}(0), v_{\lambda}(x)=0$ for all $x \in \partial B_{\lambda}$ and $v_{\lambda}(0)=\lambda \cdot v_{1}(0)>0, \lambda>0$. If $\lambda>0$ is sufficiently small the graph of $v_{\lambda}$ lies below the graph of $u$ in the ball $B_{\lambda}(0)$. Now let $\lambda$ increase monotonically up to a certain value $\lambda_{0}$ until points of contact $\left(y_{0}, u_{0}\right), u_{0}:=u\left(y_{0}\right)$, first appear. Denote by

$$
\mathcal{T}:=\left\{x \in \overline{B_{\lambda_{0}}}(0) ; u(x)=v_{\lambda_{0}}(x)\right\}
$$

the closed "touching set" of $v_{\lambda_{0}}$ and $u$ (note that $v_{\lambda_{0}} \leq u$ on $\overline{B_{\lambda_{0}}(0)}$ ). Since $|D u|$ is bounded in $\overline{B_{\lambda_{0}}(0)}$ and $\left|D v_{\lambda_{0}}(x)\right| \rightarrow \infty$ as $x \rightarrow \partial B_{\lambda_{0}}(0), \mathcal{T}$ must be contained in the interior of $B_{\lambda_{0}}(0)$. Denote by $B_{\varepsilon}$ an open ball with the properties

(a) $\overline{B_{\varepsilon}} \subset B_{\lambda_{0}}$ (0) and

(b) $B_{\varepsilon} \cap \mathcal{T}=\emptyset, \partial B_{\varepsilon} \cap \mathcal{T} \neq \emptyset$.

By relation (1.1) we have on $\overline{B_{\varepsilon}}$

$$
\Delta u-\frac{D_{i} u D_{j} u D_{i j} u}{1+|D u|^{2}}-\frac{\alpha}{u} \leq 0,
$$

and by Theorem 2.1,

$$
\Delta v_{\lambda_{0}}-\frac{D_{i} v_{\lambda_{0}} D_{j} v_{\lambda_{0}} D_{i j} v_{\lambda_{0}}}{\left(1+\left|D v_{\lambda_{0}}\right|^{2}\right)}-\frac{\alpha}{v_{\lambda_{0}}}=0
$$


By a standard device invoking the mean value theorem, we obtain for the difference $w:=$ $v_{\lambda_{0}}-u$ the elliptic inequality

$$
L w=a_{i j}(x) D_{i j} w+b_{i} D_{i} w+c w \geq 0 \text { in } B_{\varepsilon},
$$

with suitable coefficients $a_{i j}, b_{i}$ and $c:=\alpha\left(u v_{\lambda_{0}}\right)^{-1}<0$. Concluding we have $w<0$ on $B_{\varepsilon}$ and $w\left(z_{0}\right)=0$ for any point $z_{0} \in \partial B_{\varepsilon} \cap \mathcal{T}$. Also, since each $z_{0} \in \mathcal{T}$ is a point of tangency for both surfaces $u$ and $v_{\lambda_{0}}$, we must have $D w\left(z_{0}\right)=0$. This however contradicts E. Hopf's boundary point Lemma, according to which the outer normal derivative of $w$ at a boundary maximum must be positive. Theorem 1.1 is completely proved.

Proof of Theorem 1.5 The proof is similar, using that the submanifold $M$ has no boundary in $\mathbb{R}^{n} \times \mathbb{R}_{0}^{+}$. Indeed, suppose on the contrary the existence of a stationary submanifold $M$ with empty boundary, then we could find some number $\lambda_{0}>0$ such that the solution $v_{\lambda_{0}}$ touches $M$ at some interior point, again an obvious contradiction to Hopfs' boundary point lemma.

We now turn to the

Proof of Theorem 1.6 Let $u, v \in C^{2}(\Omega) \cap C^{0}(\bar{\Omega})$ and put

$$
Q(u):=\Delta u-\frac{D_{i} u D_{j} u}{1+|D u|^{2}} D_{i j} u-\frac{\alpha}{u},
$$

then by the maximum principle (see for example Gilbarg-Trudinger [15], chapters 3 and 10.1) we have the following comparison and uniqueness result:

$$
\begin{aligned}
& \text { Suppose } Q(u) \geq Q(v) \text { in } \Omega \text { and } u \leq v \text { on } \partial \Omega \text { then } u \leq v \text { in } \Omega \text {; } \\
& \text { if in addition } u\left(x_{0}\right)=v\left(x_{0}\right) \text { at some point } x_{0} \in \Omega \text { then } u \equiv v \text { in } \Omega .
\end{aligned}
$$

Hence the solutions $v_{\lambda} \in C^{2}\left(B_{\lambda}(0)\right) \cap C^{0}\left(\overline{B_{\lambda}(0)}\right), \lambda>0$, obtained in Theorem 2.1 may be used as barriers to conclude the a priori $L_{\infty}$-bound

$$
0<c(K, \Omega, \alpha, n) \leq u(x) \leq C(\Omega, \alpha, n)
$$

for all $x \in K$, any compact set $K \subset \Omega$, any solution $u$ of (1.2) and constants $c \leq C$ depending only on the quantities indicated.

For $\varepsilon>0$ consider the approximate problems

$$
\left\{\begin{array}{l}
Q\left(u^{\varepsilon}\right)=0 \text { in } \Omega, \quad u^{\varepsilon}>0 \text { in } \Omega, \text { and } \\
u^{\varepsilon}=\varepsilon \text { on } \partial \Omega .
\end{array}\right.
$$

By the a priori bound (3.9) and classical Schauder theory (see for example [15] chapter 1015 , in particular Corollary 14.7 , where - in this special case - the mean convexity condition of $\partial \Omega$ is required) we obtain a unique $C^{2}(\Omega) \cap C^{0}(\bar{\Omega})$ solution $u^{\varepsilon}$ of (3.10) satisfying the Hölder norm estimate

$$
\left\|u^{\varepsilon}\right\|_{2, \beta, K} \leq C(K, \alpha, n, \Omega)
$$

for all $\varepsilon \in(0,1)$, all compact subsets $K \subset \Omega$ and some $\beta>0$.

The maximum principle (M) implies that $u^{\varepsilon}$ are monotonic in $\varepsilon$, whence

$$
u(x):=\lim _{\varepsilon \searrow 0} u^{\varepsilon}(x)
$$


exists pointwise in $\Omega$. By (3.9), (3.10) and (3.11) $u$ is a positive solution of

$$
Q(u)=0 \text { in } \Omega \text {. }
$$

Finally, let $0<\varepsilon_{1} \leq \varepsilon_{2}<1$, then by (M) also $u_{\varepsilon_{1}} \leq u_{\varepsilon_{2}}$ in $\bar{\Omega}$. On the other hand we have the inequality

$$
Q\left(u^{\varepsilon_{1}}+\left(\varepsilon_{2}-\varepsilon_{1}\right)\right)=\alpha\left(\varepsilon_{2}-\varepsilon_{1}\right)\left(u^{\varepsilon_{1}}\left(u^{\varepsilon_{1}}+\varepsilon_{2}-\varepsilon_{1}\right)\right)^{-1} \leq 0
$$

so we infer -again by the maximum principle (M)- that also

$$
u^{\varepsilon_{2}} \leq u^{\varepsilon_{1}}+\left(\varepsilon_{2}-\varepsilon_{1}\right) \text { in } \bar{\Omega}
$$

whence the convergence in (3.12) is uniform. In particular $u \in C^{0}(\bar{\Omega}) \cap C^{2}(\Omega)$ and $u=0$ on $\partial \Omega$. Uniqueness follows similarly.

\section{References}

1. Anderson, M.T.: Complete minimal varieties in hyperbolic space. Invent. Math. 69(3), 477-494 (1982)

2. Bemelmans, J., Dierkes, U.: On a singular variational integral with linear growth. I. Existence and regularity of minimizers. Arch. Ration. Mech. Anal. 100(1), 83-103 (1987)

3. Bernstein, S.: Sur les surfaces définies au moyen de leur courbure moyenne ou totale. Ann. Sci. École Norm. Sup. (3) 27, 233-256 (1910)

4. Bombieri, E., De Giorgi, E., Giusti, E.: Minimal cones and the Bernstein problem. Invent. Math. 7, 243-268 (1969)

5. Dierkes, U.: A geometric maximum principle, Plateau's problem for surfaces of prescribed mean curvature, and the two-dimensional analogue of the catenary. In: Partial Differential Equations and Calculus of Variations, vol. 1357 of Lecture Notes in Math. pp. 116-141. Springer, Berlin (1988)

6. Dierkes, U.: Minimal hypercones and $C^{0,1 / 2}$ minimizers for a singular variational problem. Indiana Univ. Math. J. 37(4), 841-863 (1988)

7. Dierkes, U.: A Bernstein result for energy minimizing hypersurfaces. Calc. Var. Partial Differ. Equ. 1(1), 37-54 (1993)

8. Dierkes, U.: Curvature estimates for minimal hypersurfaces in singular spaces. Invent. Math. 122(3), 453-473 (1995)

9. Dierkes, U.: On the regularity of solutions for a singular variational problem. Math. Z. 225(4), 657-670 (1997)

10. Dierkes, U., Hildebrandt, S., Tromba, A.J.: Global Analysis of Minimal Surfaces, 2nd edn. vol. 341 of Grundlehren der Mathematischen Wissenschaften. Springer, Heidelberg (2010)

11. Dierkes, U., Huisken, G.: The $n$-dimensional analogue of the catenary: existence and nonexistence. Pac. J. Math. 141(1), 47-54 (1990)

12. Dierkes, U., Tennstädt, T.: Bernstein results for symmetric minimal surfaces of controlled growth. Ann. Sci. Norm. Super. Pisa XVIII, 4 (2018)

13. Finn, R.: Remarks relevant to minimal surfaces, and to surfaces of prescribed mean curvature. J. Anal. Math. 14, 139-160 (1965)

14. Finn, R.: On solutions of mean curvature type inequalities. In: Partial Differential Equations and Applications, vol. 177 of Lecture Notes in Pure and Appl. Math. pp. 147-153. Dekker, New York (1996)

15. Gilbarg, D., Trudinger, N.S.: Elliptic Partial Differential Equations of Second Order, 2nd edn. vol. 224 of Grundlehren der Mathematischen Wissenschaften. Springer, Heidelberg (1998)

16. Heinz, E.: über Flächen mit eineindeutiger Projektion auf eine Ebene, deren Krümmungen durch Ungleichungen eingeschränkt sind. Math. Ann. 129, 451-454 (1955)

17. Keiper, J.B.: The axially symmetric n-tectum (1980)

18. Lin, F.-H.: On the Dirichlet problem for minimal graphs in hyperbolic space. Invent. Math. 96(3), 593-612 (1989)

19. López, R.: Invariant singular minimal surfaces. Ann. Glob. Anal. Geom. 1, 75 (2018)

20. Usami, H.: Nonexistence of positive entire solutions for elliptic inequalities of the mean curvature type. J. Differ. Equ. 111(2), 472-480 (1994) 